\title{
Peptides of Aminoxy Acids: A Molecular Dynamics Simulation Study of Conformational Equilibria under Various Conditions
}

\author{
Christine Peter, Xavier Daura, and Wilfred F. van Gunsteren* \\ Contribution from the Laboratory of Physical Chemistry, Swiss Federal Institute of Technology Zürich, \\ ETH-Zentrum, CH-8092 Zürich, Switzerland
}

\begin{abstract}
Molecular dynamics simulations have been performed to study the conformational behavior of peptide analogues formed by $\alpha$-aminoxy acids. The influence of temperature, type of solvent, and chain length has been investigated. The NMR and CD spectra of these peptides, as well as ab initio quantum-mechanical calculations, indicate the presence of strong intramolecular hydrogen bonds between adjacent residues. In the simulations in chloroform the eight-membered-ring strucures formed by these hydrogen bonds were frequently observed, leading to a very stable $1.8_{8}$-helix both for a tri- and a tetramer, in good agreement with experimental results. In water those secondary structure elements were broken up, and completely different parts of conformational space were sampled. The conformational distributions in chloroform and water show only a small overlap. This illustrates the importance of treating solvent degrees of freedom explicitly especially in biomolecular simulations.
\end{abstract}

\section{Introduction}

Some nonnatural peptide analogues, e.g. $\beta$-peptides, have turned out to be important model compounds in biomolecular simulations as they adopt a large variety of comparatively stable secondary structures even in the case of relatively short chains. The general aim is to get insight into the mechanism of structure formation and folding of polypeptides by investigating equilibrium properties and folding pathways of such compounds with the help of molecular dynamics (MD) simulations.

Peptides of $\alpha$-aminoxy acids are oxa-analogues of $\beta$-peptides where an oxygen atom replaces the $\beta$-carbon atom. They are also peptidomimetics and form very stable turn and helix structures and are, therefore, of both theoretical and pharmaceutical interest. It has been found that in homochiral oxapeptides a strong eight-membered-ring structure appears that is built up by a hydrogen bond between adjacent aminoxy-acid residues. ${ }^{1}$ This so-called $\mathrm{N}-\mathrm{O}$ turn is very similar to the $\gamma$-turn in normal peptides. Consecutive $\mathrm{N}-\mathrm{O}$ turns in aminoxy-acid oligomers lead to formation of a 1.88-helix which could already be observed for tripeptides in chloroform solution. ${ }^{2}$ Thus the number of residues that is required in oxa-peptides for formation of a helical structure is much smaller than that in normal peptides, and even smaller than that in $\beta$-peptides.

In this paper we present MD simulation studies on the conformational behavior of aminoxy-acid peptides as a function of peptide length, type of solvent, and temperature to point out the influence of these factors on secondary structure formation and stability.

\section{Results and Discussion}

The compounds chosen for this investigation were the (S)aminoxy-acid tripeptide and tetrapeptide that are shown in

* Address correspondence to this author.

(1) Yang, D.; Ng, F.-F.; Li, Z.-J.; Wu, Y.-D.; Chan, K. W.; Wang, D.P. J. Am. Chem. Soc. 1996, 118, 9794-9795.

(2) Yang, D.; Qu, J.; Li, B.; Ng, F.-F.; Wang, X.-C.; Cheung, K.-K.; Wang, D.-P.; Wu, Y.-D. J. Am. Chem. Soc. 1999, 121, 589-590.
A<smiles>CCC(C)C(ON(I)C(=O)C(ON(C)C(=O)C(C)(C)C)C(C)C)C(=O)NOC(C(=O)OC(C)(C)C)C(C)C</smiles>

1- $\alpha \mathrm{O}$-VAL --- 2- $\alpha \mathrm{O}-\mathrm{ILE}$--- 3- $\alpha \mathrm{O}-\mathrm{VAL}$

B

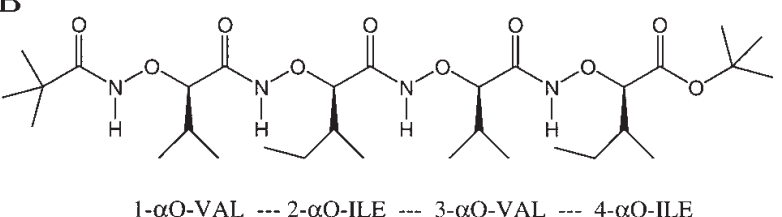

C

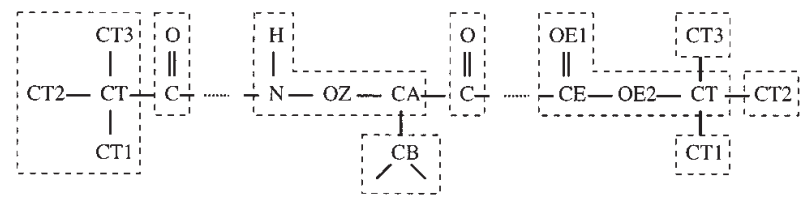

Figure 1. Chemical formula of the aminoxy-acid tripeptide (A) and tetrapeptide (B) and atom names (C) used in the definition of the force field parameters in Table 4 . The dashed lines indicate so-called charge groups of the GROMOS96 force field. ${ }^{8}$

Figure 1. Simulations of the tripeptide were performed in chloroform at 293 and $340 \mathrm{~K}$ (indicated as Tri $293 \mathrm{c}$ and $\mathrm{Tri}_{340 \mathrm{c}}$ ) and in water at 300 and $340 \mathrm{~K}$ (indicated as Tri $300 \mathrm{w}$ and $\mathrm{Tri}_{340 \mathrm{w}}$ ). The tetrapeptide was simulated in chloroform at $293 \mathrm{~K}$ (indicated as $\mathrm{Tet}_{293 \mathrm{c}}$ ). In all cases the starting conformation was an extended chain. Table 1 gives an overview of the five simulations. They were analyzed by performing a cluster analysis using the atom-positional root-mean-square distance (RMSD) as similarity criterion; ${ }^{3}$ the numbering of the clusters is according to their relative population: cluster 1 is most populated, etc. 


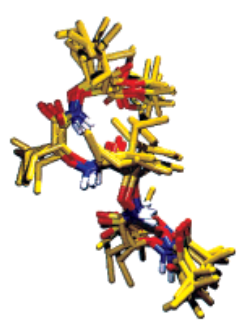

A

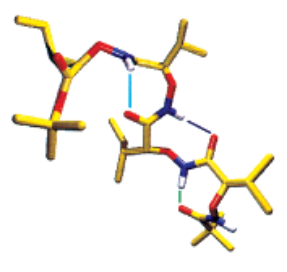

B

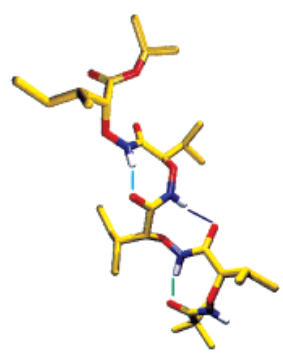

C

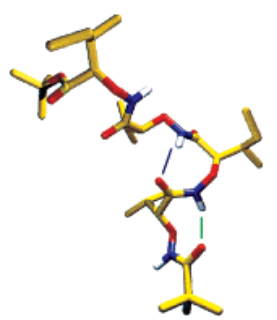

D

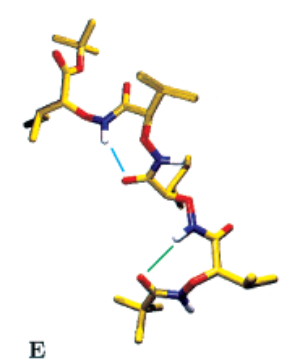

E

Figure 2. Structures of some central cluster members of the simulation of the tetramer in chloroform at $293 \mathrm{~K}\left(\mathrm{Tet}_{293 \mathrm{c}}\right.$ ) with the hydrogen bonds indicated: $\mathrm{HB}_{8-1}$ (green); $\mathrm{HB}_{8-2}$ (blue); $\mathrm{HB}_{8-3}$ (cyan). (A) Superposition of structures belonging to (the most populated) cluster 1 , (B) cluster 1 $\left(59 \% \mathrm{HB}_{8-1}, 47 \% \mathrm{HB}_{8-2}, 25 \% \mathrm{HB}_{8-3}\right)$, (C) cluster $2\left(62 \% \mathrm{HB}_{8-1}, 57 \% \mathrm{HB}_{8-2}, 55 \% \mathrm{HB}_{8-3}\right)$, (D) cluster $11\left(36 \% \mathrm{HB}_{8-1}, 40 \% \mathrm{HB}_{8-2}, 0 \% \mathrm{HB}_{8-3}\right)$, and (E) cluster $14\left(42 \% \mathrm{HB}_{8-1}, 0 \% \mathrm{HB}_{8-2}, 40 \% \mathrm{HB}_{8-3}\right)$.

Table 1. Overview of the MD Simulations of the Peptides of $\alpha$-Aminoxy Acids (the length is the effective length after skipping the first ns of equilibration)

\begin{tabular}{ccclcc}
\hline label & $\begin{array}{c}\text { no. of } \\
\text { residues }\end{array}$ & temp [K] & solvent & $\begin{array}{c}\text { no. of solvent } \\
\text { molecules }\end{array}$ & length [ns] \\
\hline $\mathrm{Tet}_{293 \mathrm{c}}$ & 4 & 293 & $\mathrm{CHCl}_{3}$ & 828 & 49 \\
$\mathrm{Tri}_{293 \mathrm{c}}$ & 3 & 293 & $\mathrm{CHCl}_{3}$ & 672 & 72 \\
$\mathrm{Tri}_{340 \mathrm{c}}$ & 3 & 340 & $\mathrm{CHCl}_{3}$ & 672 & 72 \\
$\mathrm{Tri}_{300 \mathrm{w}}$ & 3 & 300 & $\mathrm{H}_{2} \mathrm{O}$ & 2290 & 25 \\
$\mathrm{Tri}_{340 \mathrm{w}}$ & 3 & 340 & $\mathrm{H}_{2} \mathrm{O}$ & 2290 & 25 \\
\hline
\end{tabular}

Chloroform Simulations. In chloroform a right-handed $1.8_{8^{-}}$ helix turned out to be the dominant conformation both for the tri- and the tetrapeptide at all investigated temperatures. This helix is built up by $\mathrm{N}-\mathrm{O}$ turns, hydrogen bonds forming an eight-membered ring. In the trimer there are two possibilities to form these hydrogen bonds between adjacent aminoxy-acid residues, in the tetramer there are three. They will further on be denoted as $\mathrm{HB}_{8-1}, \mathrm{HB}_{8-2}$, and $\mathrm{HB}_{8-3}$, the numbering beginning at the N-terminal residue. In Figure 2(B and $\mathrm{C}$ ) a tetramer which forms all three possible $\mathrm{N}-\mathrm{O}$ turns can be seen. In the simulations another type of hydrogen bond is observed that leads to a 12 -membered-ring structure $\left(\mathrm{HB}_{12-1}\right)$, which is shown in Figure 3E. The structures that appear in MD simulations are in good agreement with low energy conformations from ab initio quantum-mechanical calculations, ${ }^{4}$ where the right-handed $1.8_{8}$-helix was found to be the structure with lowest energy for all investigated chain lengths. The 12membered ring was also found, ${ }^{4}$ but only in energetically less favored conformers. Two other possible types of secondary structures which were not observed in MD simulations are the left-handed $1.8_{8}$-helix and a $\beta$-turn-like structure with two $\mathrm{N}-\mathrm{O}$ turns pointing into the opposite direction. In ab initio calculations the left-handed 1.8 -helix is not at all a low energy conformation for $(S)$-oxa-polypeptides, and the $\beta$-turn-like structure is less favored than the right-handed $1.8_{8}$-helix.

In Figure $3(\mathrm{~A}-\mathrm{E})$ some representative structures for the simulations of the trimer in chloroform can be seen. At both temperatures the two most populated clusters are $1.8_{8}$-helices, the major difference between the two clusters being the position of the C-terminal residue, which is not fixed by a hydrogen bond. Only examples of structures from simulation $\mathrm{Tri}_{293 \mathrm{c}}$ are shown, since those from simulation $\mathrm{Tri}_{340 \mathrm{c}}$ are in principle the same. An overview of the fraction of structures in each simulation that show one or the other type of hydrogen bond is given in Table 2. It is obvious that the $\mathrm{N}-\mathrm{O}$ turns with $\mathrm{HB}_{8-1}$

(3) Daura, X.; van Gunsteren, W. F.; Mark, A. E. Proteins 1999, 34, 269-280.

(4) Wu, Y.-D.; Wang, D.-P.; Chan, K. W. K.; Yang, D. J. Am. Chem. Soc. 1999, 121, 11189-11196.

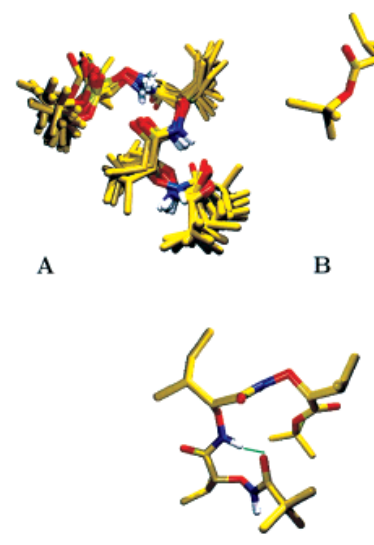

D

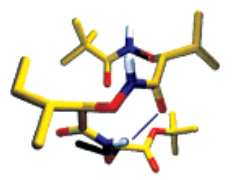

$\mathbf{F}$

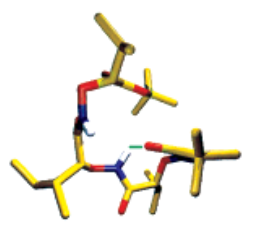

H

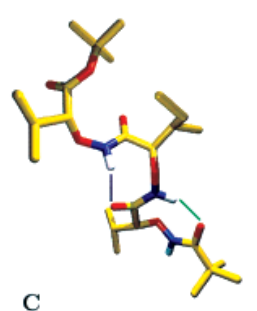

C

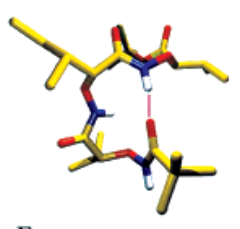

E

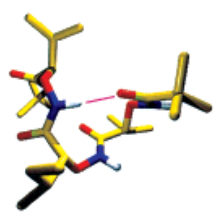

G

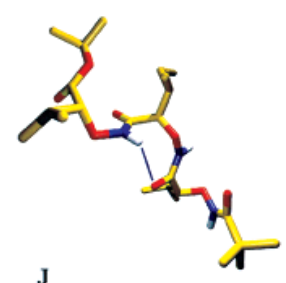

Figure 3. Structures of some central cluster members of the simulations of the trimer in different solvents and at different temperatures with the hydrogen bonds indicated: $\mathrm{HB}_{8-1}$ (green), $\mathrm{HB}_{8-2}$ (blue), $\mathrm{HB}_{12-1}$ (magenta). (A) Superposition of structures belonging to cluster 1 in $\mathrm{Tri}_{293}$, (B) $\mathrm{Tri}_{293 \mathrm{c}}$ cluster 1 (68\% HB $8-1,22 \% \mathrm{HB}_{8-2}, 13 \%$ both), (C) $\mathrm{Tri}_{293 \mathrm{c}}$ cluster $2\left(54 \% \mathrm{HB}_{8-1}, 45 \% \mathrm{HB}_{8-2}, 25 \%\right.$ both), (D) Tri $293 \mathrm{c}$ cluster $11\left(62 \% \mathrm{HB}_{8-1}, 0 \% \mathrm{HB}_{8-2}\right)$, (E) $\mathrm{Tri}_{293 \mathrm{c}}$ cluster $14\left(85 \% \mathrm{HB}_{12-1}\right)$, (F) Tri $_{300 w}$ cluster $1\left(47 \% \mathrm{HB}_{8-2}\right),(\mathrm{G}) \mathrm{Tri}_{300 \mathrm{w}}$ cluster $2\left(82 \% \mathrm{HB}_{12-1}\right),(\mathrm{H})$ $\mathrm{Tri}_{300 \mathrm{w}}$ cluster $3\left(12 \% \mathrm{HB}_{8-1}\right)$, and $(\mathrm{J}) \mathrm{Tri}_{300 \mathrm{w}}$ cluster $11\left(3 \% \mathrm{HB}_{8-2}\right)$.

and $\mathrm{HB}_{8-2}$ dominate both simulations in chloroform, especially the 10 most populated clusters, in which $\mathrm{HB}_{12-1}$ does not appear at all. The fraction of structures that show $\mathrm{HB}_{8-1}$ and $\mathrm{HB}_{8-2}$ at the same time is considerably larger among the 10 most populated clusters than among those clusters that have only one member and represent less well-defined secondary structures. 
Table 2. Fraction of Hydrogen Bonds in the Various Simulations ${ }^{a}$

\begin{tabular}{|c|c|c|c|c|c|c|}
\hline & $\mathrm{H}$ bond & $\begin{array}{c}\text { Tet }_{293 \mathrm{c}} \\
{[\%]}\end{array}$ & $\begin{array}{c}\operatorname{Tri}_{293 \mathrm{c}} \\
{[\%]}\end{array}$ & $\begin{array}{c}\operatorname{Tri}_{340 \mathrm{c}} \\
{[\%]}\end{array}$ & $\begin{array}{c}\operatorname{Tri}_{300 \mathrm{w}} \\
{[\%]}\end{array}$ & $\begin{array}{c}\operatorname{Tri}_{340 \mathrm{w}} \\
{[\%]}\end{array}$ \\
\hline \multirow[t]{7}{*}{ A } & $\mathrm{HB}_{8-1}$ & 47 & 46 & 42 & 4 & 6 \\
\hline & $\mathrm{HB}_{8-2}$ & 44 & 32 & 32 & 11 & 14 \\
\hline & $\mathrm{HB}_{8-3}$ & 38 & & & & \\
\hline & $\mathrm{HB}_{12-1}$ & $<1$ & 6 & 2 & 20 & 17 \\
\hline & $\mathrm{HB}_{8-1}$ and $\mathrm{HB}_{8-2}$ & 22 & 17 & 15 & 0 & 0 \\
\hline & $\mathrm{HB}_{8-2}$ and $\mathrm{HB}_{8-3}$ & 18 & & & & \\
\hline & $\mathrm{HB}_{8-1}$ and $\mathrm{HB}_{8-3}$ & 19 & & & & \\
\hline \multirow[t]{7}{*}{ B } & $\mathrm{HB}_{8-1}$ & 55 & 50 & 48 & 2 & 3 \\
\hline & $\mathrm{HB}_{8-2}$ & 51 & 38 & 38 & 16 & 17 \\
\hline & $\mathrm{HB}_{8-3}$ & 43 & & & & \\
\hline & $\mathrm{HB}_{12-1}$ & 0 & 0 & 0 & 30 & 25 \\
\hline & $\mathrm{HB}_{8-1}$ and $\mathrm{HB}_{8-2}$ & 30 & 21 & 19 & 0 & 0 \\
\hline & $\mathrm{HB}_{8-2}$ and $\mathrm{HB}_{8-3}$ & 22 & & & & \\
\hline & $\mathrm{HB}_{8-1}$ and $\mathrm{HB}_{8-3}$ & 25 & & & & \\
\hline \multirow[t]{7}{*}{$\mathrm{C}$} & $\mathrm{HB}_{8-1}$ & 34 & 34 & 28 & 9 & 8 \\
\hline & $\mathrm{HB}_{8-2}$ & 32 & 22 & 20 & 5 & 11 \\
\hline & $\mathrm{HB}_{8-3}$ & 30 & & & & \\
\hline & $\mathrm{HB}_{12-1}$ & 1 & 5 & 3 & 7 & 4 \\
\hline & $\mathrm{HB}_{8-1}$ and $\mathrm{HB}_{8-2}$ & 14 & 4 & 6 & 0 & 1 \\
\hline & $\mathrm{HB}_{8-2}$ and $\mathrm{HB}_{8-3}$ & 9 & & & & \\
\hline & $\mathrm{HB}_{8-1}$ and $\mathrm{HB}_{8-3}$ & 7 & & & & \\
\hline
\end{tabular}

${ }^{a} \mathrm{~A}$, all structures; B, structures of clusters 1-10; C, structures of

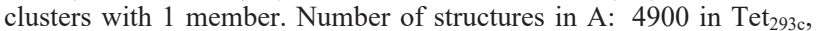
7200 in $\operatorname{Tri}_{293 \mathrm{c}}$ and $\mathrm{Tri}_{340 \mathrm{c}}, 2500$ in $\mathrm{Tri}_{300 \mathrm{w}}$ and $\mathrm{Tri}_{340 \mathrm{w}}$. Number of structures in B: 2490 in $\mathrm{Tet}_{293 \mathrm{c}}, 2934$ in $\mathrm{Tri}_{293 \mathrm{c}}, 2599$ in $\mathrm{Tri}_{340 \mathrm{c}}, 1147$ in $\operatorname{Tri}_{300 \mathrm{w}}, 1017$ in $\mathrm{Tri}_{340 \mathrm{w}}$. Number of structures in C: 97 in Tet $293 \mathrm{c}$, 241 in $\operatorname{Tri}_{293 c}, 394$ in Tri 340 c, 179 in Tri ${ }_{300 w}, 303$ in $\operatorname{Tri}_{340 w}$

One can also see that $\mathrm{HB}_{12-1}$ is less often formed at the higher temperature as one would expect for entropic reasons.

In Figure 4 the backbone atom-positional root-mean-square distance (RMSD) of the structures in the simulations (taken every $0.05 \mathrm{~ns}$ ) from some representative cluster members is presented together with the observed hydrogen bonds. In Figure 4A fluctuations between clusters 1 and 2 can be observed for almost the whole simulation period, except for some intermissions where the 12-membered ring (e.g. cluster 14) is formed. There is no single, longer period in which neither the helix nor the other ring structure appears, which would be characterized by a large RMSD from cluster 1 and a lack of hydrogen bonding and which could be called an "unfolding event". So, these secondary structures seem to be extremely stable in chloroform solution. The results for the simulation at $340 \mathrm{~K}$ are very similar. As one can see in Figure 4B, the higher temperature does not destabilize the helix. Only the lifetimes of clusters 1 and 2 are shorter, which implies more frequent transitions between them through to a rotation of the $\mathrm{C}$-terminus.

In the tetramer the 12 -membered ring appears very rarely $\left(\mathrm{HB}_{12-1}\right.$ is found in less than $1 \%$ of all structures, Table 2$)$, possibly because there are cooperativity effects that favor the helix with three $\mathrm{N}-\mathrm{O}$ turns in a line. ${ }^{4}$ The fraction of structures with $\mathrm{N}-\mathrm{O}$ turns is even higher than that for the trimer, especially those that form more than one of the hydrogen bonds at the same time. Figure 2 shows some characteristic conformations of the tetramer. As in the simulations of the trimer, clusters 1 and 2 represent the $1.8_{8}$-helix, with the orientation of the $\mathrm{C}$-terminal residue as the major difference (Figure 2, B and C). Other typical structures are helices with one of the three hydrogen bonds broken (Figure 2, C and D). The secondary structure of the tetramer is remarkably stable, and no complete "unfolding" was observed in the simulations as one can see in Figure 5 where neither the backbone-atom-positional RMSD from the helical conformation nor the hydrogen bonding indicates a complete breaking of the helix.
A
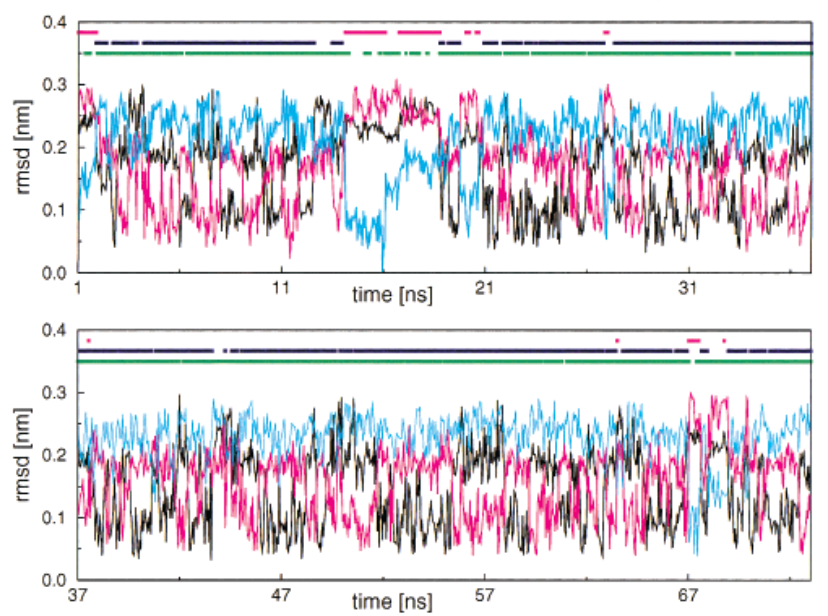

B
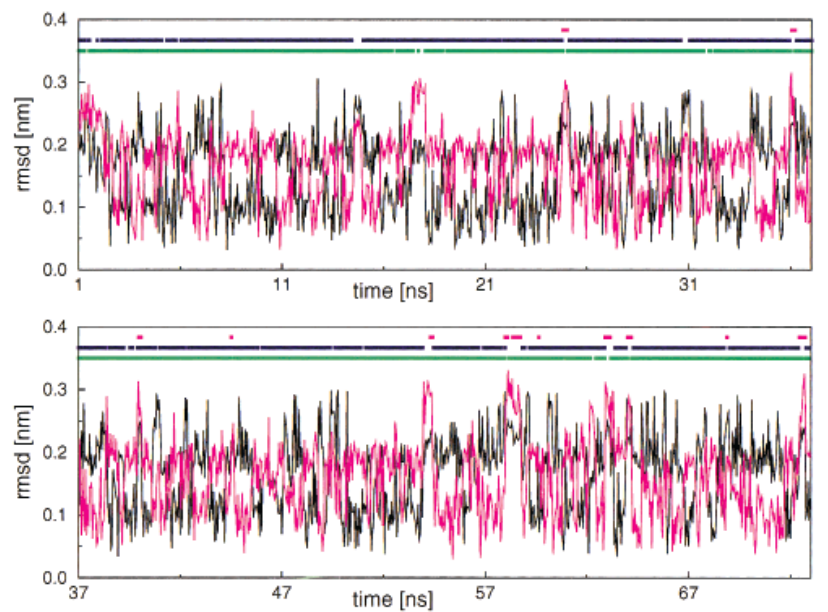

Figure 4. Backbone atom-positional root-mean-square distance (RMSD) of structures in the simulations (taken every $0.05 \mathrm{~ns}$ ) of the trimer in chloroform from several typical clusters (conformations): (A) $\operatorname{Tri}_{293 \mathrm{c}}$ cluster 1 (black), cluster 2 (magenta), cluster 14 (cyan); (B) $\operatorname{Tri}_{340 \mathrm{c}}$ cluster 1 (black), cluster 2 (magenta). Hydrogen bond monitoring: $\mathrm{HB}_{8-1}$ (green), $\mathrm{HB}_{8-2}$ (blue), $\mathrm{HB}_{12-1}$ (magenta).

Water Simulations. In water the pattern of secondary structures looks completely different. Due to hydrogen bonding to water molecules the intramolecular hydrogen bonds are less favorable. In Figure $3(\mathrm{~F}-\mathrm{J})$ typical structures of clusters in water are shown. The 1.88-helix with two simultaneous hydrogen bonds is not found at all, but there are clusters that contain structures with one of the three already mentioned hydrogen bonds $\mathrm{HB}_{8-1}, \mathrm{HB}_{8-2}$, or $\mathrm{HB}_{12-1}$ (Table 2). Other hydrogen bonds are possible, but they were not observed with significant frequency in the simulations. Most of the conformations are coil-like, but there are also clusters with an extended structure, as shown in Figure 3J. A stable secondary structure cannot be detected. As in the chloroform simulations, the predominant types of structures at the two temperatures do not differ much, only the lifetime of the conformations is shorter at the higher temperature. As is demonstrated by the RMSD plot in Figure 6 , the molecule stays for shorter time periods in an extended conformation at the higher temperature. The hydrogen bond statistics are given in Table 2. Compared to the simulations in chloroform the 12-membered ring appears more frequently, as 

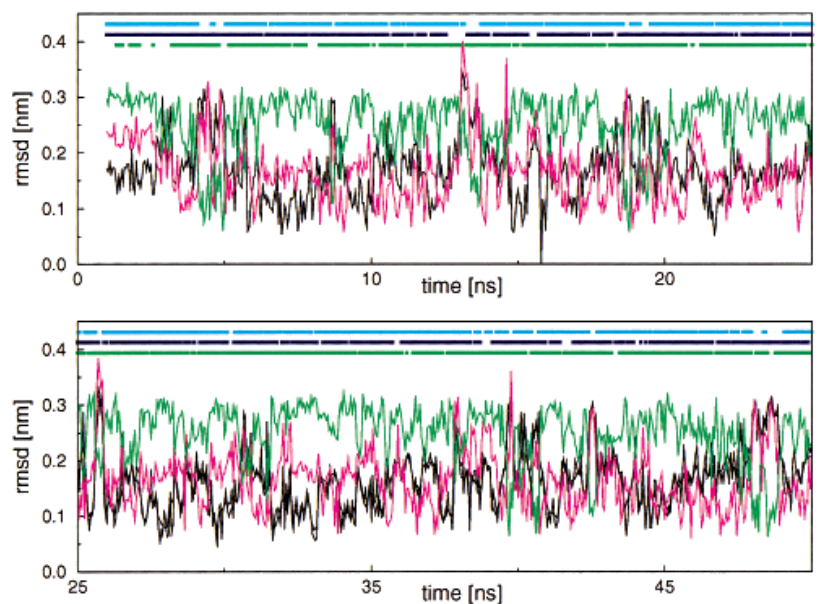

Figure 5. Backbone atom-positional root-mean-square distance (RMSD) of structures in the simulation (taken every $0.05 \mathrm{~ns}$ ) of the tetramer in chloroform at $293 \mathrm{~K}$ (Tet ${ }_{293 \mathrm{c}}$ ) from cluster 1 (black), cluster 2 (magenta), and cluster 11 (green). Hydrogen bond monitoring: $\mathrm{HB}_{8-1}$ (green); $\mathrm{HB}_{8-2}$ (blue); $\mathrm{HB}_{8-3}$ (cyan).
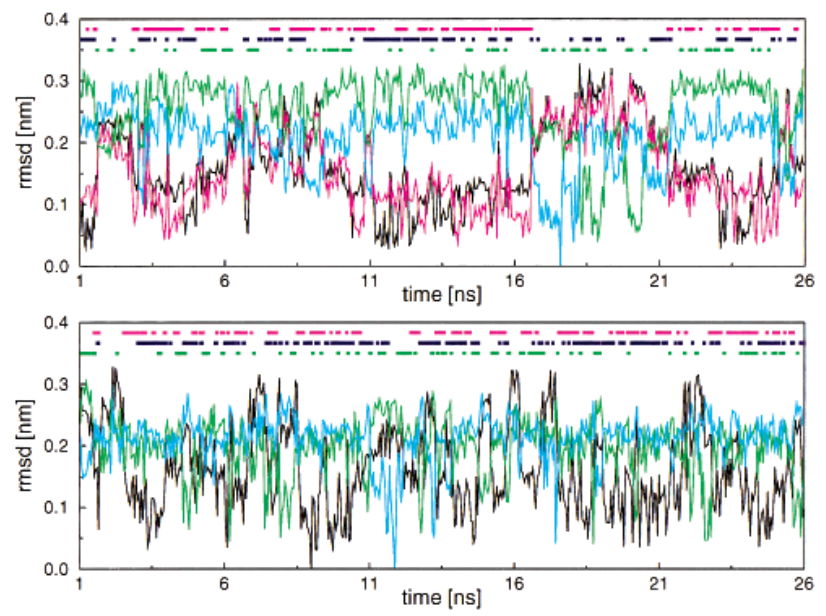

Figure 6. Backbone atom-positional root-mean-square distance (RMSD) of structures in simulations (taken every $0.05 \mathrm{~ns}$ ) of the trimer in $\mathrm{H}_{2} \mathrm{O}$ from several typical clusters (e, extended; c, coil): (upper panel) Tri $300 \mathrm{w}$ cluster 1 (black; c), cluster 2 (magenta; c), cluster 11 (green; e), cluster 13 (cyan; e); (lower panel) Tri ${ }_{340 \mathrm{w}}$ cluster 1 (black; c), cluster 8 (green; e), cluster 15 (cyan; e). Hydrogen bond monitoring: $\mathrm{HB}_{8-1}$ (green), $\mathrm{HB}_{8-2}$ (blue), $\mathrm{HB}_{12-1}$ (magenta).

the fact that only one intramolecular hydrogen bond can be formed in this conformation plays no role in water. Short-range (along the backbone) intramolecular hydrogen bonding seems to be slightly favored at the higher temperature, as is expected for entropic reasons in particular regarding the water molecules.

Comparison of the Simulations. Figure 7 shows the relative presence of the clusters in the five simulations up to cluster number 100. Comparing Tri $293 \mathrm{c}$ and $\mathrm{Tri}_{340 \mathrm{c}}$ or Tri $\mathrm{Ti}_{300 \mathrm{w}}$ and $\mathrm{Tri}_{340 \mathrm{w}}$ one can see that at the higher temperatures the relative weight of the most populated clusters is lower. The increase of the absolute number of clusters with temperature is $46 \%$ for the chloroform simulations and $45 \%$ for the water simulations. The fraction of structures that belong to the first 100 clusters is 7 and $8 \%$ lower at the higher temperature for chloroform and water, respectively. This indicates that there is a solvent independent influence of temperature on the rate of sampling new structures instead of resampling low-energy conformations. For the tetramer the relative weight of the first clusters is
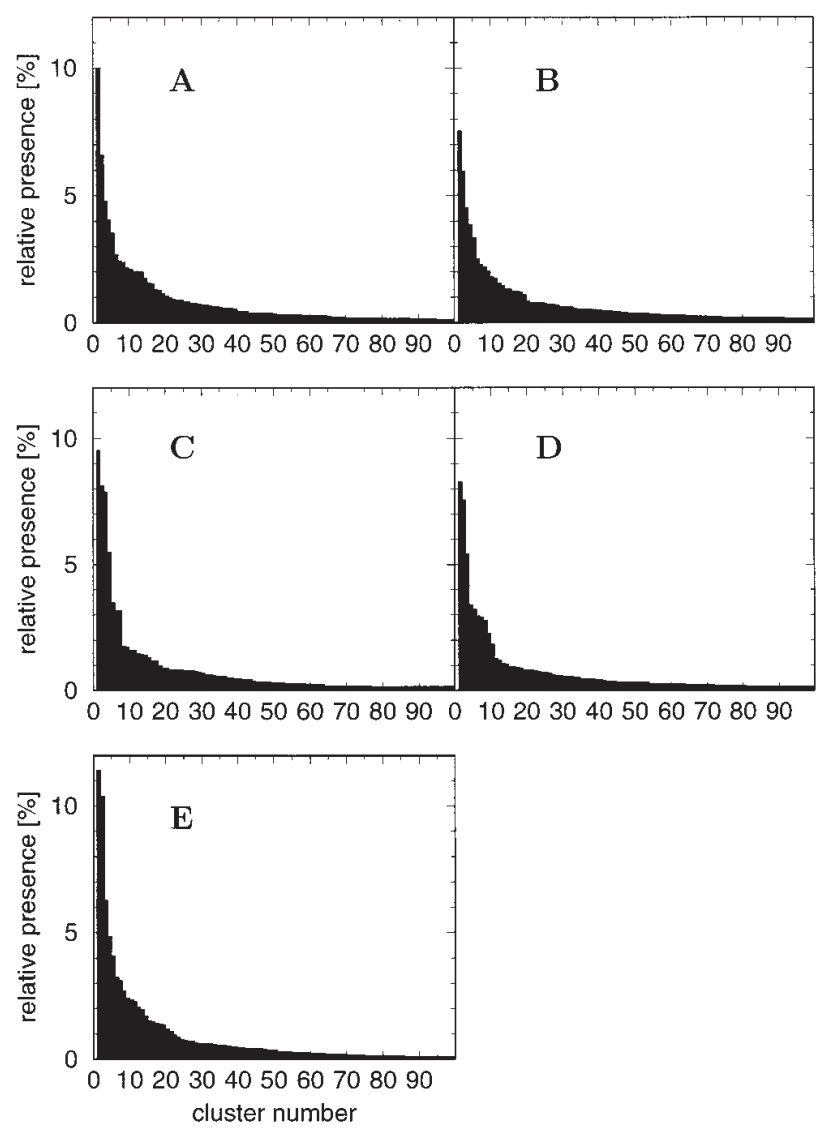

Figure 7. Relative presence of the various clusters (conformations) in the five simulations (in brackets: simulation length; total number of clusters; fraction of structures in the first 100 clusters): (A) Tri ${ }_{293}$ c (72 ns; 529; 85\%); (B) Tri $340 \mathrm{c}(72 \mathrm{~ns} ; 770 ; 78 \%$ ); (C) Tri $300 \mathrm{w}$ (25 ns; $355 ; 85 \%$ ); (D) Tri $_{340 \mathrm{w}}(25 \mathrm{~ns} ; 515 ; 77 \%$ ); and (E) Tet $293 \mathrm{c}$ (49 ns; 266; $94 \%)$.

especially high (Figure 7E). This confirms the higher stability of the helix for longer oligomers.

To compare how the peptides explore conformational space in the different simulations, a cluster analysis of concatenated trajectories was performed. We produced the following four new trajectories: Tri $\mathrm{i}_{\mathrm{T}}$ (low temperature) was made by appending Tri $_{300 \mathrm{w}}$ to $\mathrm{Tri}_{293 \mathrm{c}}$ and $\mathrm{Tri}_{\mathrm{ht}}$ (high temperature) by appending $\mathrm{Tri}_{340 \mathrm{w}}$ to $\mathrm{Tri}_{340 \mathrm{c}}$ to investigate the influence of the solvent at the two temperatures, Tri $_{\mathrm{c}}$ (chloroform) was made by appending $\operatorname{Tri}_{340 \mathrm{c}}$ to $\mathrm{Tri}_{293 \mathrm{c}}$ and $\mathrm{Tri}_{\mathrm{w}}$ (water) by appending $\mathrm{Tri}_{340 \mathrm{w}}$ to $\mathrm{Tri}_{300 \mathrm{w}}$ to investigate the influence of the temperature for the two solvents. For $\operatorname{Tri}_{l \mathrm{~T}}$ and $\mathrm{Tri}_{\mathrm{hT}}$ the clustering was done for different lengths of the trajectory (see Figure 8). After 72 ns when the solvent changes from chloroform to water, there is a sudden jump in the total number of clusters and also in the number of clusters with more than 5 or less than 6 member-structures. This shows that the structures that are added here do not fit into already existing clusters, that is, the conformations in water differ from the conformations in chloroform. In Figure 8 one can also see that the number of clusters per nanosecond of trajectory increases faster at the higher temperatures which means that the exploration of conformational space is faster. The clustering of the concatenated trajectories was analyzed more closely by counting all clusters that are significantly populated by structures from both parts of the combined trajectory. A cluster with significant contribution from both parts of the trajectory was defined by having a fraction of more than 15 and less than $85 \%$ of its structures from each part, where 


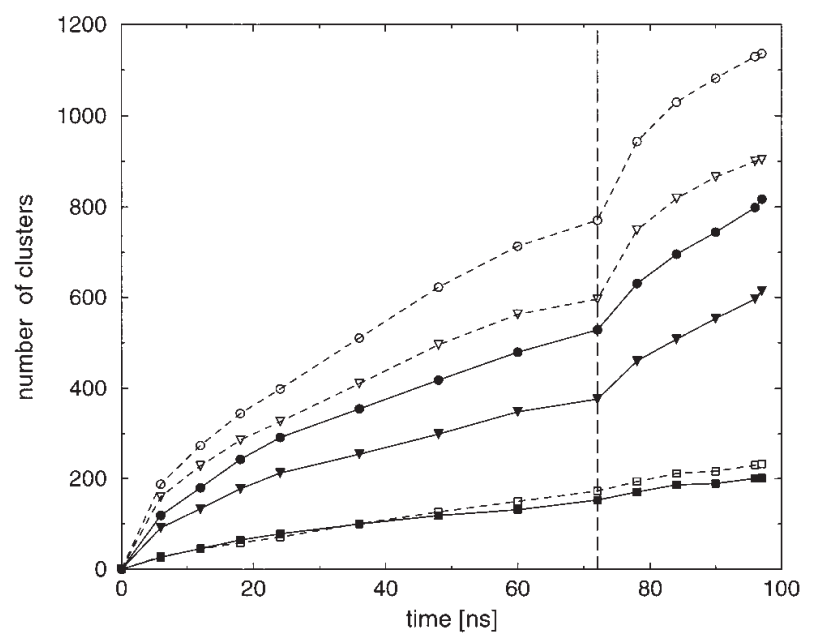

Figure 8. Number of clusters as a function of trajectory length. Solid lines/solid symbols: $\operatorname{Tri}_{\mathrm{TT}}\left(=\operatorname{Tri}_{340 \mathrm{c}}(72 \mathrm{~ns})\right.$ with $\operatorname{Tri}_{300 \mathrm{w}}(25 \mathrm{~ns})$ appended). Dashed lines/open symbols: $\operatorname{Tri}_{\mathrm{hT}}\left(=\operatorname{Tri}_{340 \mathrm{c}}(72 \mathrm{~ns})\right.$ with Tri $340 \mathrm{w}$ (25 ns) appended). Circles: Absolute number of clusters. Triangles: Number of clusters with more than five members in the concatenated trajectories. Squares: Number of clusters with less than six members in the concatenated trajectories. Vertical dashed line: Change of solvent from chloroform to water in the concatenated trajectories.

the different time lengths of the trajectory parts were of course taken into account. Thus, for $\operatorname{Tri}_{1 \mathrm{~T}} 71$ out of 817 clusters, that is $9 \%$, and for $\operatorname{Tri}_{\mathrm{hT}} 110$ out of 1136 clusters, that is $10 \%$, are "mixed" clusters. That this criterion makes sense can be seen from the difference between the total number of clusters of the two single trajectories before merging and that of the concatenated one, which is 67 in the case of Tri $\mathrm{T}_{\mathrm{IT}}$ and 149 in the case of $\operatorname{Tri}_{\mathrm{hT}}$. So, at both temperatures the conformational space of the trimer visited in chloroform has only a limited overlap with the one visited in water. The same analysis was done to determine the overlap between the conformational spaces visited in a single solvent at two temperatures. In chloroform $\left(\mathrm{Tri}_{\mathrm{c}}\right)$ 320 out of 954 clusters, that is $34 \%$, are "mixed" clusters, while in water $\left(\operatorname{Tri}_{\mathrm{W}}\right) 137$ out of 708 clusters, that is $19 \%$, are "mixed" clusters. Although the conformational overlap of two trajectories with different solvents is very small, two trajectories with the same solvent but at different temperatures seem to sample more similar regions of conformational space. This picture is confirmed and the difference between the effect of changing solvent or temperature is enlarged, if the same analysis is done with only those clusters of the concatenated trajectories that have more than five members. In case of the concatenated trajectories $\operatorname{Tri}_{\mathrm{c}}$ and $\operatorname{Tri}_{\mathrm{w}} 86$ and $90 \%$ of the clusters with more than five members are populated by structures from both parts, i.e., both temperatures. For $\operatorname{Tri}_{I \mathrm{~T}}$ and $\operatorname{Tri}_{\mathrm{hT}} 23$ and $27 \%$ of these clusters are populated by structures from both parts, i.e., both solvents.

Comparison with Experiment: NOE Distances. The main experimental evidence for the existence of a helical structure of aminoxy-acid peptides in chloroform solution comes from ${ }^{1}$ H-ROESY NMR spectra. ${ }^{2}$ The nuclear Overhauser effects (NOEs) between $\mathrm{NH}_{i}$ and $\mathrm{C}_{\alpha} \mathrm{H}_{i}$ are strong, while those between $\mathrm{NH}_{i+1}$ and $\mathrm{C}_{\alpha} \mathrm{H}_{i}$ are weak, thus indicating that, on average, the distance between $\mathrm{NH}_{i}$ and $\mathrm{C}_{\alpha} \mathrm{H}_{i}$ is much shorter than the distance between $\mathrm{NH}_{i+1}$ and $\mathrm{C}_{\alpha} \mathrm{H}_{i}$. The average interproton distances in the simulations were calculated for comparison. This was done by using $r^{-6}$-averaging, which is the method that corresponds best with the time averaging in the NMR experiment in the case of a small molecule which is tumbling fast compared to
Table 3. Averages of Interproton (indices denote residue number) Distances Calculated from the Simulation To Compare with NOE Values $^{a}$

\begin{tabular}{cccccccc}
\hline & & \multicolumn{5}{c}{$\mathrm{v}_{6}[\mathrm{~nm}]$} & \\
\cline { 2 - 6 } \multicolumn{2}{c}{ H atoms } & Tet $_{293 \mathrm{c}}$ & $\operatorname{Tri}_{293 \mathrm{c}}$ & $\operatorname{Tri}_{340 \mathrm{c}}$ & $\operatorname{Tri}_{300 \mathrm{w}}$ & $\operatorname{Tri}_{340 \mathrm{w}}$ & NOE \\
\hline $\mathrm{NH}_{1}$ & $\mathrm{C}_{\alpha} \mathrm{H}_{1}$ & 0.245 & 0.241 & 0.240 & 0.235 & 0.234 & $\mathrm{~s}$ \\
$\mathrm{NH}_{2}$ & $\mathrm{C}_{\alpha} \mathrm{H}_{1}$ & 0.268 & 0.281 & 0.277 & 0.230 & 0.228 & $\mathrm{w}$ \\
$\mathrm{NH}_{2}$ & $\mathrm{C}_{\alpha} \mathrm{H}_{2}$ & 0.233 & 0.236 & 0.234 & 0.231 & 0.229 & $\mathrm{~s}$ \\
$\mathrm{NH}_{3}$ & $\mathrm{C}_{\alpha} \mathrm{H}_{2}$ & 0.287 & 0.277 & 0.285 & 0.239 & 0.239 & $\mathrm{w}$ \\
$\mathrm{NH}_{3}$ & $\mathrm{C}_{\alpha} \mathrm{H}_{3}$ & 0.232 & 0.238 & 0.236 & 0.233 & 0.228 & $\mathrm{~S}$ \\
$\mathrm{NH}_{4}$ & $\mathrm{C}_{\alpha} \mathrm{H}_{3}$ & 0.295 & & & & & $\mathrm{w}$ \\
$\mathrm{NH}_{4}$ & $\mathrm{C}_{\alpha} \mathrm{H}_{4}$ & 0.238 & & & & & $\mathrm{~s}$ \\
\hline
\end{tabular}

${ }^{a} v_{6}=\left\langle r^{-6}\right\rangle^{-1 / 6}$. Last column: relative intensity of the NOEs (w: weak; s: strong) from experiments in chloroform. ${ }^{2}$ The values for the tetrapeptide were obtained from Dr. Dan Yang (private communication).

internal motions. ${ }^{5,6}$ The results are presented in Table 3. In all chloroform simulations the average distance between $\mathrm{NH}_{i}$ and $\mathrm{C}_{\alpha} \mathrm{H}_{i}$ is indeed $0.04-0.05 \mathrm{~nm}$ shorter than the distance between $\mathrm{NH}_{i+1}$ and $\mathrm{C}_{\alpha} \mathrm{H}_{i}$, except at the $\mathrm{N}$-terminus of the tetrapeptide. In the water simulations, where no helical conformation could be detected and coil conformations are dominant, this difference does not appear, and all NOEs should accordingly have similar intensities.

\section{Conclusions}

The conformational behavior of short peptides of $\alpha$-aminoxy acids has been studied by MD simulations at two temperatures $(300$ and $340 \mathrm{~K})$ in two different solvents (chloroform and water) and for two chain lengths (a trimer and a tetramer). NMR experiments and CD spectra indicate that these peptides adopt a helical conformation in chloroform, and from ab initio quantum-mechanical calculations a right-handed $1.8_{8}$-helix was predicted as the most stable secondary structure. The simulations in chloroform using the GROMOS96 force field show that this helix is indeed formed after starting from an extended conformation and that it is very stable even at high temperatures. The data from the NMR experiments are satisfied. The predicted cooperativity effect which favors formation of subsequent eightmembered hydrogen-bonded rings and results in an even more stable helix for longer oligomers could also be observed.

The MD simulations once more point out the importance of an explicit treatment of solvent degrees of freedom, especially for biomolecules in water. The simulations in water sample a completely different part of conformational space, i.e., none of the secondary structures that are observed in chloroform simulations or that are minimum energy conformations in ab initio quantum-mechanical calculations ${ }^{4}$ turn out to be significantly present in water. This illustrates the limited applicability of ab initio quantum-mechanical methodology, in which entropic effects $(\mathrm{T}=0)$ and specific solute-solvent interactions are neglected, to highly dynamic solutes for which the structure is critically depending on the type and hydrogen-bonding properties of the solvent.

\section{Computational Methods}

Simulations. All simulations were performed using the GROMOS96 package of programs ${ }^{7,8}$ with the standard GROMOS96 43A1 united atom force field ${ }^{8}$ extended with parameters for aminoxy moieties. The

(5) Tropp, J. J. Chem. Phys 1980, 72, 6035-6043.

(6) Daura, X.; Antes, I.; van Gunsteren, W. F.; Thiel, W.; Mark, A. E. Proteins 1999, 36, 542-555.

(7) Scott, W. R. P.; Hünenberger, P. H.; Tironi, I. G.; Mark, A. E.; Billeter, S. R.; Fennen, J.; Torda, A. E.; Huber, T.; Krüger, P.; van Gunsteren, W. F. J. Phys. Chem. A 1999, 103, 3596-3607. 
Table 4. Force Field Parameters for the Backbone Atoms in Aminoxy-Acid Peptides and for the Ester Group at the C-terminus ${ }^{a}$

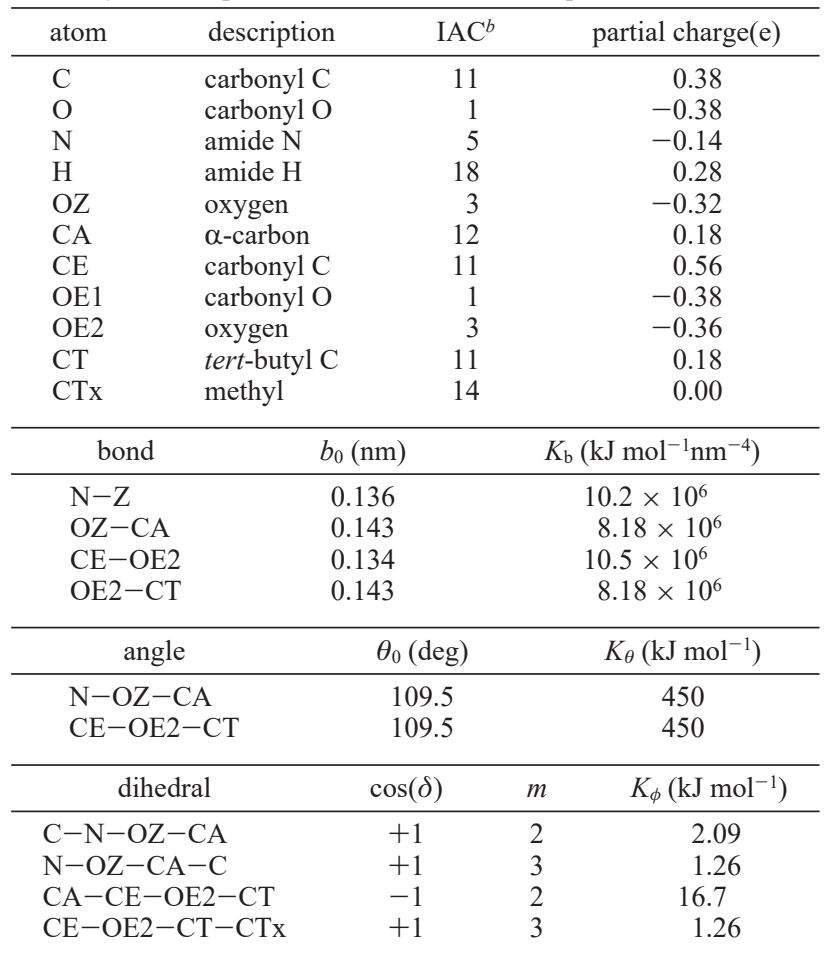

${ }_{a}$ The atom names are defined in Figure 1; parameters (bond lengths, angles, and dihedrals) of carbonyl, amide and tert-butyl groups (not given) were chosen in analogy to GROMOS96 force field parameters for peptides and non-carbohydrates. ${ }^{b}$ GROMOS 96 integer atom code, describes the Lennard-Jones parameters of the corresponding atoms.

$\alpha$-aminoxy-acid molecular topology building blocks were constructed in analogy to the corresponding $\alpha$-amino acid ones of the GROMOS96 force field. ${ }^{8}$ The parameters affected by the insertion of the extra oxygen atom in the backbone are reported in Table 4 . They were chosen based on ab initio results obtained with the Gaussian 94 program using the $\mathrm{HF} / 6-31 \mathrm{G}^{*}$ method.

Initially, the solute was placed in an extended starting conformation (with all backbone torsional angles in trans) at the center of a truncated octahedron with a minimum distance of the solute atoms to the box wall of $1.8 \mathrm{~nm}$. This box was filled with solvent molecules such that the distance between (non-hydrogen) solvent and solute atoms was bigger than $0.23 \mathrm{~nm}$. The resulting numbers of solvent molecules are given in Table 1. The chloroform model was taken from Tironi et al. ${ }^{9}$

(8) Van Gunsteren, W. F.; Billeter, S. R.; Eising, A. A.; Hünenberger, P. H.; Krüger, P.; Mark, A. E.; Scott, W. R. P.; Tironi, I. G. Biomolecular Simulation: The GROMOS96 Manual and User Guide; Vdf Hochschulverlag AG an der ETH Zürich: Zürich 1996. and for water the SPC model ${ }^{10}$ was used. In all five simulations periodic boundary conditions were applied.

To relax the system a steepest descent energy minimization was initially performed. The MD simulations were started by taking the initial velocities from a Maxwellian distribution at the corresponding temperature. Solvent and solute were independently weakly coupled to a temperature bath with a relaxation time of $0.1 \mathrm{ps} .{ }^{11}$ The systems were also coupled to a pressure bath at $1 \mathrm{~atm}$ with a relaxation time of $0.2 \mathrm{ps}$, and an isothermal compressibility of $1.0 \times 10^{-3}\left(\mathrm{~kJ} \mathrm{~mol}^{-1} \mathrm{~nm}^{-3}\right)^{-1}$ for the chloroform simulations, and a relaxation time of $0.5 \mathrm{ps}$, and an isothermal compressibility of $7.0 \times 10^{-4}\left(\mathrm{~kJ} \mathrm{~mol}^{-1} \mathrm{~nm}^{-3}\right)^{-1}$ for the water simulations. Bond lengths were constrained using the SHAKE algorithm with a geometric tolerance of $10^{-4},{ }^{12}$ so that the time step for the leapfrog integration scheme could be set to $0.002 \mathrm{ps}$. For the nonbonded interactions a triple-range method with cutoff radii of 0.8 and $1.4 \mathrm{~nm}$ was used. ${ }^{7,8}$ Outside the longer cutoff radius a reaction field approximation $^{13}$ was used with a relative dielectric permittivity of 5.0 for chloroform $^{9}$ and 54.0 for SPC water. ${ }^{14}$ The center of mass motion of the whole system was removed every 1000 time steps and the trajectory coordinates and energies were saved every $0.001 \mathrm{~ns}$ for analysis.

Analysis. A cluster analysis was done on all trajectories omitting the first nanosecond for equilibration and using the conformations every $0.01 \mathrm{~ns}$. Clustering was done as described in ref 3 by performing a rotational and translational atom-positional least-squares fit on every pair of conformations using all backbone atoms and calculating the atom-positional root-mean-square difference (RMSD) between the two structures using all backbone, carbonyl oxygen, amide hydogen, and $\beta$-carbon atoms. The similarity criterion used was an RMSD $\leq 0.07$ $\mathrm{nm}$ for the trimer and an RMSD $\leq 0.1 \mathrm{~nm}$ for the tetramer.

The criterion for a hydrogen bond in a given structure was a maximum distance of $0.25 \mathrm{~nm}$ between the hydrogen atom and the acceptor atom and a minimum angle of $135^{\circ}$ between donor, hydrogen, and acceptor.

Acknowledgment. We thank Dr. Dan Yang and co-workers for providing the ROESY spectra of the aminoxy-acid tetrapeptide. Financial support was obtained from the Schweizerischer Nationalfonds, project number 21-50929.97, which is gratefully acknowledged.

\section{JA000873T}

(9) Tironi, I. G.; van Gunsteren, W. F. Mol. Phys. 1994, 83, 381-403.

(10) Berendsen, H. J. C.; Postma, J. P. M.; van Gunsteren, W. F.; Hermans, J. In Intermolecular Forces; Pullman, B., Ed.; Reidel: Dordrecht, 1981; pp 331-342.

(11) Berendsen, H. J. C.; Postma, J. P. M.; van Gunsteren, W. F.; DiNola, A.; Haak, J. R. J. Chem. Phys. 1984, 81, 3684-3690.

(12) Ryckaert, J.-P.; Ciccotti, G.; Berendsen, H. J. C. J. Comput. Phys. 1977, 23, 327-341.

(13) Tironi, I. G.; Sperb, R.; Smith, P. E.; van Gunsteren, W. F. J. Chem. Phys. 1995, 102, 5451-5459.

(14) Smith, P. E.; van Gunsteren, W. F. J. Chem. Phys. 1994, 100, 31693174 . 